\title{
TEMPLATE GENERATION THROUGH OBJECT PROCESSING WITH SIMPLE USER INTERFACE
}

\author{
Aravind M \\ MCA Scholar,School of CS \& IT, Dept. of MCA, Jain (Deemed-to-be) University, Bangalore
}

Article DOI: https://doi.org/10.36713/epra4338

\begin{abstract}
Traditional way when an UX/UI designer needs to design a website he needs to do the rough diagram of
wireframe and then if the design proposal is been accepted by the client then the designers needs create the User
Interface of the Website should be created but what if the client has been reject the designed proposal then the
UX/UI designer need to do from scratch. Here I'm presenting you this proposal.
KEYWORDS- digital conversion, identification, recognizing, Template Generation, machine learning
\end{abstract}
\section{INTRODUCTION}

TOPUI the users can immediately draw his very own template right here a custom vision Model educated to function object recognition against HTML hand drawn patterns is used to discover significant design elements into an image, here each object will detected element based on the pattern recognition and passes through a Text Recognition Service to extract handwritten content then a valid HTML is generated as a consequence to the detected format containing the detected plan elements.

The UX/UI configuration process includes a great deal of creativity that begins a whiteboard where fashioners share thoughts. When an architect cerate's the structure of wireframe, it is generally caught inside a photo design and physically converted into some working HTML wireframe to play inside an internet browser. This takes endeavors and postpones the plan procedure. While handling this technique we face a ton of challenges, for example, Changes in the proposed plan and re-compose/Build up the HTML format, this includes a lot of time and if a structure is refactored on the whiteboard it requires some investment assets and the program reflects changes in a split second. In that sense, by method for the quit of the meeting, there is a subsequent model approved between the fashioner, designer, and buyer presenting our idea, an online arrangement that utilizes artificial intelligence to fundamentally change a written by hand UI group from a picture to a substantial HTML increase code. We should comprehend the way toward changing a manually written into a picture configuration to HTML utilizing TOPUI in more detail. Initially, the individual transfers a photo by means of the site.

At that point A tweaked creative and farsighted mannequin predicts what HTML components are available in the photograph and their zone after that manually written content center supplier peruses the content inside the imagined components. At that point of organization calculation utilizes the spatial records from all the bouncing holders of the imagined components to create a lattice shape that obliges all. At long last, A HTML age motor uses every one of these snippets of data to create a HTML increase code mirroring the outcome.

\section{Motivation}

- Detect Design Patterns: A Custom Vision Model trained to operate object focus in opposition to HTML hand drawn patterns is used to observe meaningful sketch elements into an image.

- Understand handwritten text: Understand handwritten text: Each detected issue is handed through a Text Recognition Service to extract handwritten content.

- Build HTML: A valid HTML is generated as an outcome to the detected structure containing the detected sketch elements.

\section{LITERATURE REVIEW}

[1] In this paper the creator cited that the paper gives a total Optical Character Acknowledgment (OCR) framework for computerized of an image in a camera caught picture/illustrations inserted printed documents for handheld gadgets. From the outset, literary 


\section{SJIF Impact Factor: 6.260| ISI I.F.Value:1.241| Journal DOI: 10.36713/epra2016 ISSN: 2455-7838(Online) EPRA International Journal of Research and Development (IJRD) \\ Volume: 5 | Issue: 4 | April 2020 - Peer Reviewed Journal}

substance zones are extricated and slant rectified. At that point, these territories are binarized and portioned into follows and characters. Characters are passed into the acknowledgment module.

[2] In this paper the writer cited that a total OCR philosophy for perceiving memorable reports, both printed or manually written other than to know-how to aptitude of the textual style, is introduced. This technique comprises of three stages: The initial two stages allude to building up a database for instruction the utilization of a lot of archives, while the $1 / 3$ one alludes to acknowledgment of new record pictures. Initial, a pre-preparing step that comprises of photograph binarization and upgrade happens. At a second step a zenith - down division technique is utilized so as to watch printed content lines, expressions and characters. A grouping plan is then embraced so as to group characters of practically identical structure.

\section{PROPOSED SYSTEM}

OCR is a wide locale of research inside PC Vision, two of its basic issues are acknowledgment of characters from machine printed (or) written by hand records and Archive Structure Investigation (DSA). In the two territories, no longer exclusively is established researchers performing progresses anyway moreover programming program businesses are exceptionally vivacious on becoming acquainted with about this theme. Maybe, the most interesting improvements have been made in organization's business programming. Here is the stream outline it shows the total progression of distinguishing the articles in an arrangement way and checks the character at that point slant the remedy make the divisions of the characters lastly it perceiving the characters in structure words. The primary problem is respected explained when discussing machine printed composing, and primarily understood when talking about manually written prepared frameworks cognizance. By utilizing this OCR strategy here I have made the venture where the client gives the contribution as a picture design and by doling out some watchword in the string, for example, "Btn for Catch, Lbl for Name, Txt for Textbox, and so on." similarly it recognizes the catchphrase a produces the HTML Code in the wake of getting the code of the yield at long last he will test the code in the editorial manager and he can alter if any progressions expected to him in like manner.

\section{PROBLEM STATEMENT}

Traditional way the UX/UI designer needs to design a website he needs to do the rough diagram of wireframe and then if the design proposal is been accepted by the client then the designers needs create the User Interface of the Website should be created but when the client has been reject the designed proposal then the UX/UI designer need to do from scratch.

\section{PROBLEM DEFINITION}

OCR is a wide area of research inside PC Vision, two of its basic issues are acknowledgment of characters from machine printed (or) written by hand records and Report Structure Examination (DSA).

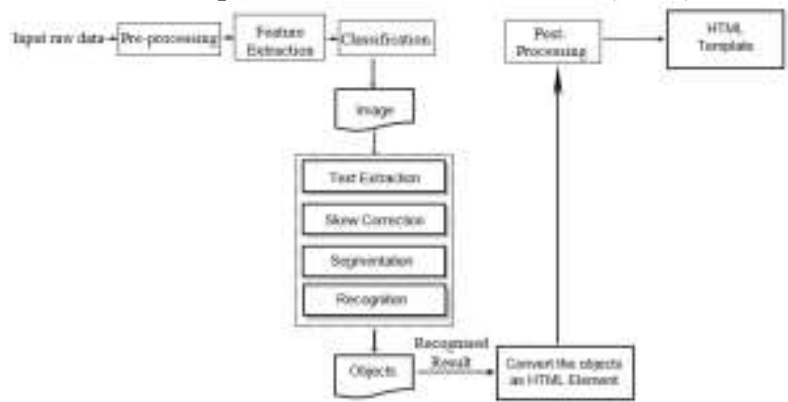

Figure 1: OCR Text Recognition Pattern

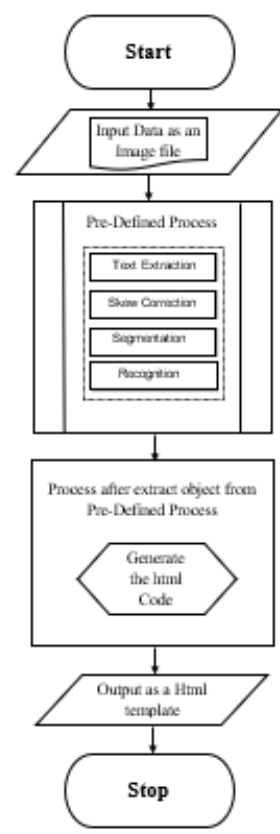

Figure 2: Flow chart

In the two territories, no longer exclusively is established researchers performing progresses anyway moreover programming program businesses are exceptionally lively on becoming more acquainted with about this theme. Maybe, the most intriguing improvements have been made in enterprise's business programming. Here is the stream graph in figure- 1 it shows the total progression of distinguishing the items in a succession way and checks the character at that point slant the rectification make the divisions of the characters lastly it perceiving the characters in structure words as appeared in figure-3 


\section{EPRA International Journal of Research and Development (IJRD)}

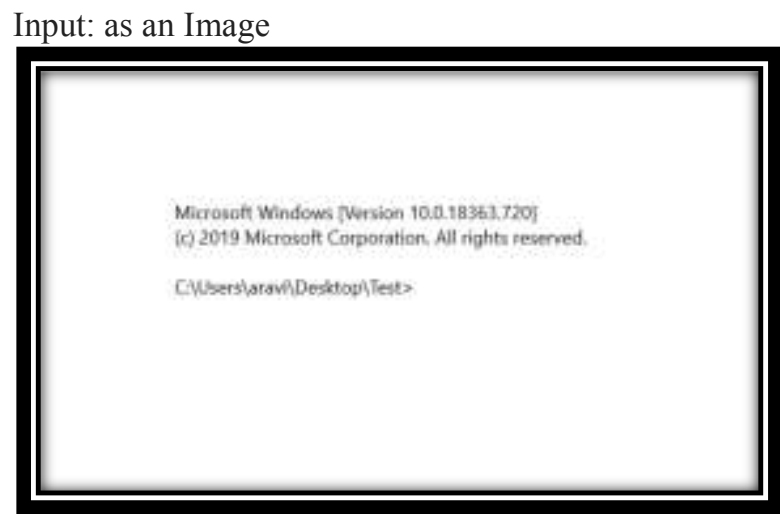

Figure 3: Sample Image as an input

Result:

Windows PowerShell
Copyright (C) Microsoft Corporation. All
rights reserved.
PS C:IUserslaravilDesktop\Test>

The primary issue is respected understood when discussing machine printed composing, and chiefly settled when talking about manually written prepared frameworks cognizance. By utilizing this OCR technique here I have made the venture where the client gives the contribution as a picture group appeared in figure- 4 and by doling out some catchphrase in the string, for example, "Btn for Catch, Lbl for Name, Txt for Textbox, and so forth." like insightful it distinguishes the watchword a produces the HTML Code appeared in figure-5 in the wake of getting the code of the yield at last he will test the code in the supervisor and he can adjust if any progressions expected to him in like manner as appeared in the figure-6.

Input: as image

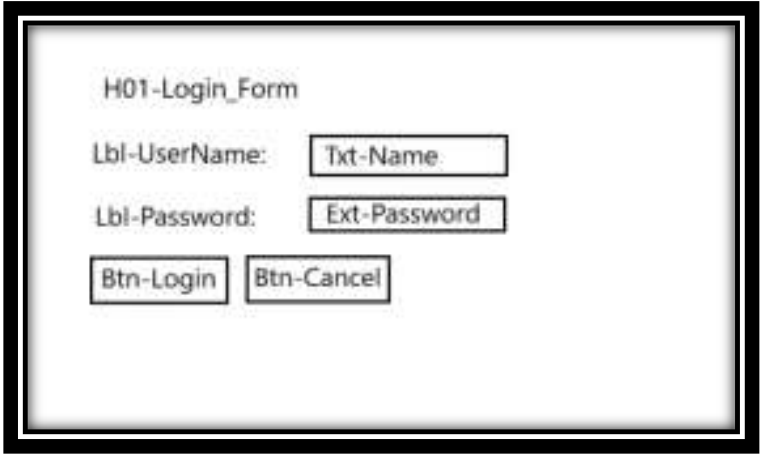

Figure 4
Output:

Code:

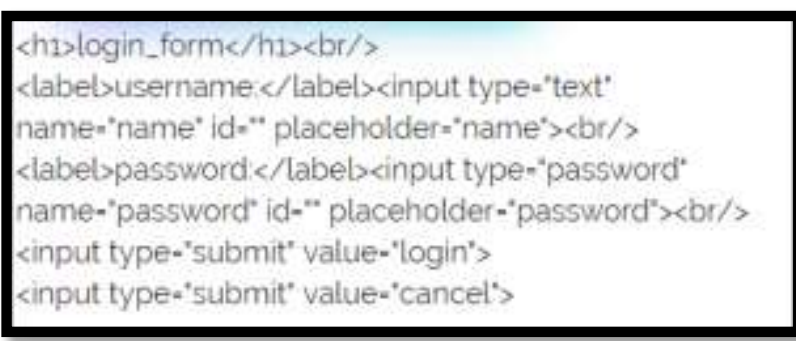

Html-Template:

Figure 5

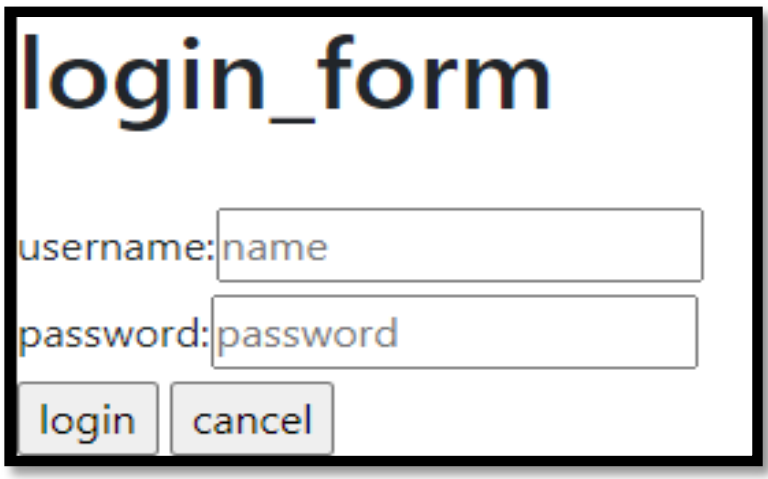

Figure 6

\section{CONCLUSION}

In this task we've utilized python-based free and open source web-application structure, which follows the Model Layout View (MTV) compositional example. this undertaking we have remembered for picture process with highlight extraction and content location highlights, here the client will be giving the contribution as a picture and our web-application will process the given information and creates the code then this web-application will give to the client.

\section{REFERENCES}

[1] F. M. Ayatullah, M. Nabamita, B. Subhadip and N. Mita, "Design of an Optical Character Recognition System for Camera-based Handheld Devices," IJCSI International Journal of Computer Science Issues, pp. 283-289, 2011.

[2] V. G, G. B, S. N and P. S.J, "A Complete Optical Character Recognition Methodology for Historical Documents," The Eighth IAPR International Workshop on Document Analysis Systems, pp. 525-532, 2008. 